International Journal of Applied Mathematical Research, $5(4)(2016)$ 187-191
International Journal of Applied Mathematical Research
WPC
Website: www.sciencepubco.com/index.php/IJAMR
doi: $10.14419 /$ ijamr.v5i4.6174
Research paper

\title{
Solving linear two-dimensional Fredholm integral equations system by triangular functions
}

\author{
Elias Hengamian Asl ${ }^{1}$ and Jafar Saberi-Nadjafi ${ }^{1 *}$ \\ ${ }^{1}$ Department of Applied Mathematics, Ferdowsi University of Mashhad, Mashhad, Iran \\ *Corresponding author E-mail: najafi141@gmail.com
}

\begin{abstract}
In this paper, we intend to offer a numerical method to solve linear two-dimensional Fredholm integral equations system of the second kind. This method converts the given two-dimensional Fredholm integral equations system into a linear system of algebraic equations by using two-dimensional triangular functions. Moreover, we prove the convergence of the method. Finally the proposed method is illustrated by two examples and also results are compared with the exact solution by using computer simulations.
\end{abstract}

Keywords: Two-dimensional Fredholm integral equations system of the second kind (2D-FIES-2); Two m-sets of one-dimensional triangular functions (1D-TFs); Two-dimensional triangular functions (2D-TFs)

\section{Introduction}

It is well known that the differential and integral equations are one of the important parts of the analysis theory that play major role in numerical analysis. There are many numerical methods which have been focusing on the solution of integral equations. For example, Tricomi, in his book [11], introduced the classical method of successive approximations for nonlinear integral equations. Variational iteration method [10] was effective and convenient for solving integral equations. Some numerical methods have been investigated to solve linear Fredholm integral equations of the second kind in two-dimensional space [12]. The set of triangular orthogonal functions (TFs) has been presented and applied to analysis of dynamical systems. These functions was first treated by Deb et al $[5,6]$. Recently, introduced triangular orthogonal functions have been applied for solving Volterra Fredholm integral equation and integral equation system by Babolian et al. [2,4] and Almasieh et al. [1]. Maleknejad et al. [8] have used two-dimensional triangular functions for solving nonlinear class of mixed Volterra Fredholm integral equations. Also, Mirzaee et al. [9] have used triangular functions for solving the two-dimensional fuzzy Fredholm integral equations of the second kind and Hengamian Asl [7] have used triangular functions for solving the one-dimensional fuzzy Fredholm integral equations system.

The aim of this paper is to apply the two-dimensional triangular functions (2D-TFs) for the linear two-dimensional Fredholm integral equations system of the second kind (2D-FIES-2). We show that, the proposed method is well performs for linear 2D-FIES-2.

This paper is organized as follows. Review of triangular functions and their properties which will be used later, is briefly provided in Section 2. Section 3 presents a numerical method for solving system of two-dimensional Fredholm integral equations of the second kind. Convergence analysis for the method is established in Section 4.
Finally, we illustrate in Section 5 some numerical examples to show the efficiency and accuracy of the proposed method.

\section{Review of triangular functions}

\subsection{One-dimensional triangular functions}

Definition 2.1: [5]Two m-sets of one-dimensional triangular functions (1D-TFs) are defined over the interval [0,T] as:

$T 1_{i}(t)=\left\{\begin{array}{cc}1-\frac{t-i h}{h}, & i h \leq t<(i+1) h, \\ 0, & o . w,\end{array}\right.$
$T 2_{i}(t)=\left\{\begin{array}{cc}\frac{t-i h}{h}, & i h \leq t<(i+1) h, \\ 0, & o . w,\end{array}\right.$

where $i=0,1, \cdots, m-1, h=\frac{T}{m}$, with a positive integer value for $m$. In this paper, it is assumed that $T=1$. Also, we have:

$\int_{0}^{1} T 1_{i}(t) T 1_{j}(t) \mathrm{d} t=\int_{0}^{1} T 2_{i}(t) T 2_{j}(t) \mathrm{d} t= \begin{cases}\frac{h}{3}, & i=j \\ 0, & i \neq j\end{cases}$

$\int_{0}^{1} T 1_{i}(t) T 2_{j}(t) \mathrm{d} t=\int_{0}^{1} T 2_{i}(t) T 1_{j}(t) \mathrm{d} t= \begin{cases}\frac{h}{6}, & i=j, \\ 0, & i \neq j .\end{cases}$

\subsection{Two-dimensional triangular functions}

Definition 2.2: [5] An $\left(m_{1} \times m_{2}\right)$-set of two-dimensional triangular functions $(2 D-T F s)$ are defined on $\Omega=[0,1] \times[0,1]$ as: 
$T_{i, j}^{1,1}(s, t)=\left\{\begin{array}{cc}\left(1-\frac{s-i h_{1}}{h_{1}}\right)\left(1-\frac{t-j h_{2}}{h_{2}}\right), & i h_{1} \leq s<(i+1) h_{1}, \\ 0, & j h_{2} \leq t<(j+1) h_{2}, \\ \text { otherwise }\end{array}\right.$

$T_{i, j}^{1,2}(s, t)=\left\{\begin{array}{cc}\left(1-\frac{s-i h_{1}}{h_{1}}\right)\left(\frac{t-j h_{2}}{h_{2}}\right), & i h_{1} \leq s<(i+1) h_{1}, \\ 0, & j h_{2} \leq t<(j+1) h_{2}, \\ \text { otherwise }\end{array}\right.$

$T_{i, j}^{2,1}(s, t)=\left\{\begin{array}{cc}\left(\frac{s-i h_{1}}{h_{1}}\right)\left(1-\frac{t-j h_{2}}{h_{2}}\right), & i h_{1} \leq s<(i+1) h_{1}, \\ 0, & j h_{2} \leq t<(j+1) h_{2}, \\ \text { otherwise },\end{array}\right.$

$T_{i, j}^{2,2}(s, t)=\left\{\begin{array}{cc}\left(\frac{s-i h_{1}}{h_{1}}\right)\left(\frac{t-j h_{2}}{h_{2}}\right), & i h_{1} \leq s<(i+1) h_{1}, \\ 0, & j h_{2} \leq t<(j+1) h_{2}, \\ \text { otherwise }\end{array}\right.$

where $i=0,1, \cdots, m_{1}-1, j=0,1, \cdots, m_{2}-1, h_{1}=\frac{1}{m_{1}}, h_{2}=\frac{1}{m_{2}}$. $m_{1}$ and $m_{2}$ are arbitrary positive integers. It is clear that

$T_{i, j}^{1,1}(s, t)=T 1_{i}(s) \cdot T 1_{j}(t)$

$T_{i, j}^{1,2}(s, t)=T 1_{i}(s) \cdot T 2_{j}(t)$,

$T_{i, j}^{2,1}(s, t)=T 2_{i}(s) \cdot T 1_{j}(t)$,

$T_{i, j}^{2,2}(s, t)=T 2_{i}(s) \cdot T 2_{j}(t)$,

From Eq.s (1), (2) and (3) we have:

$\int_{0}^{1} \int_{0}^{1} T_{i_{1}, j_{1}}^{p_{1}, q_{1}}(s, t) \cdot T_{i_{2}, j_{2}}^{p_{2}, q_{2}}(s, t) \mathrm{d} s \mathrm{~d} t=\Delta_{p_{1}, p_{2}} \delta_{i_{1}, i_{2}} \cdot \Delta_{q_{1}, q_{2}} \delta_{j_{1}, j_{2}}$,

where $\delta$ denotes the Kronecker delta function and

$\Delta_{\alpha, \beta}=\left\{\begin{array}{rrr}\frac{h}{3}, & \alpha=\beta \in\{1,2\}, \\ \frac{h}{6}, & \alpha \neq \beta .\end{array}\right.$

On the other hand, if

$T 11(s, t)=\left[T_{0,0}^{1,1}(s, t), \ldots, T_{0, m_{2}-1}^{1,1}, T_{1,0}^{1,1}(s, t), \ldots, T_{m_{1}-1, m_{2}-1}^{1,1}(s, t)\right]^{T}$, $T 12(s, t)=\left[T_{0,0}^{1,2}(s, t), \ldots, T_{0, m_{2}-1}^{1,2}, T_{1,0}^{1,2}(s, t), \ldots, T_{m_{1}-1, m_{2}-1}^{1,2}(s, t)\right]^{T}$, $T 21(s, t)=\left[T_{0,0}^{2,1}(s, t), \ldots, T_{0, m_{2}-1}^{2,1}, T_{1,0}^{2,1}(s, t), \ldots, T_{m_{1}-1, m_{2}-1}^{2,1}(s, t)\right]^{T}$, $T 22(s, t)=\left[T_{0,0}^{2,2}(s, t), \ldots, T_{0, m_{2}-1}^{2,2}, T_{1,0}^{2,2}(s, t), \ldots, T_{m_{1}-1, m_{2}-1}^{2,2}(s, t)\right]^{T}$,

then $T(s, t)$, the 2D-TF vector, can be defined as follows

$T(s, t)=\left[\begin{array}{l}T 11(s, t) \\ T 12(s, t) \\ T 21(s, t) \\ T 22(s, t)\end{array}\right]_{4 m_{1} m_{2} \times 1}$

by using eq. (4), We have:

$\int_{0}^{1} \int_{0}^{1} T 11^{T}(s, t) T 11(s, t) \mathrm{d} s \mathrm{~d} t=\frac{h_{1}}{3} I_{m_{1} \times m_{1}} \otimes \frac{h_{2}}{3} I_{m_{2} \times m_{2}}$,

$\int_{0}^{1} \int_{0}^{1} T 11^{T}(s, t) T 12(s, t) \mathrm{d} s \mathrm{~d} t=\frac{h_{1}}{3} I_{m_{1} \times m_{1}} \otimes \frac{h_{2}}{6} I_{m_{2} \times m_{2}}$,

$\int_{0}^{1} \int_{0}^{1} T 11^{T}(s, t) T 21(s, t) \mathrm{d} s \mathrm{~d} t=\frac{h_{1}}{6} I_{m_{1} \times m_{1}} \otimes \frac{h_{2}}{3} I_{m_{2} \times m_{2}}$,

$\int_{0}^{1} \int_{0}^{1} T 11^{T}(s, t) T 22(s, t) \mathrm{d} s \mathrm{~d} t=\frac{h_{1}}{6} I_{m_{1} \times m_{1}} \otimes \frac{h_{2}}{6} I_{m_{2} \times m_{2}}$,

where $\otimes$ denotes the Kronecker product defined for two arbitrary matrices $P$ and $Q$ as

$P \otimes Q=P_{i, j} Q$.
The same equations are implied for $T 12(s, t), T 21(s, t)$ and $T 22(s, t)$, by similar computations. Hence, we can carry out the following double integration of $T(s, t)$ :

$\int_{0}^{1} \int_{0}^{1} T^{T}(s, t) T(s, t) \mathrm{d} s \mathrm{~d} t=D$

where $D$ is $\left(4 m_{1} m_{2} \times 4 m_{1} m_{2}\right)$-matrix as follows:

$D=\left[\begin{array}{cccc}\frac{h_{1}}{3} I_{1} \otimes \frac{h_{2}}{3} I_{2} & \frac{h_{1}}{3} I_{1} \otimes \frac{h_{2}}{6} I_{2} & \frac{h_{1}}{6} I_{1} \otimes \frac{h_{2}}{3} I_{2} & \frac{h_{1}}{6} I_{1} \otimes \frac{h_{2}}{6} I_{2} \\ \frac{h_{1}}{3} I_{1} \otimes \frac{h_{2}}{6} I_{2} & \frac{h_{1}}{3} I_{1} \otimes \frac{h_{2}}{3} I_{2} & \frac{h_{1}}{6} I_{1} \otimes \frac{h_{2}}{6} I_{2} & \frac{h_{1}}{6} I_{1} \otimes \frac{h_{2}}{3} I_{2} \\ \frac{h_{1}}{6} I_{1} \otimes \frac{h_{2}}{3} I_{2} & \frac{h_{1}}{6} I_{1} \otimes \frac{h_{2}}{6} I_{2} & \frac{h_{1}}{3} I_{1} \otimes \frac{h_{2}}{3} I_{2} & \frac{h_{1}}{3} I_{1} \otimes \frac{h_{2}}{6} I_{2} \\ \frac{h_{1}}{6} I_{1} \otimes \frac{h_{2}}{6} I_{2} & \frac{h_{1}}{6} I_{1} \otimes \frac{h_{2}}{3} I_{2} & \frac{h_{1}}{3} I_{1} \otimes \frac{h_{2}}{6} I_{2} & \frac{h_{1}}{3} I_{1} \otimes \frac{h_{2}}{3} I_{2}\end{array}\right]$,

where $I_{1}=I_{m_{1} \times m_{1}}$ and $I_{2}=I_{m_{2} \times m_{2}}[8]$.

\subsection{Approximate the function with $2 \mathrm{D}$-TFs}

Let $f(s, t)$ be a function of two variables on $\Omega=[0,1] \times[0,1]$. It can be approximated with respect to $2 \mathrm{D}-\mathrm{TFs}$ as follows:

$$
\begin{aligned}
f(s, t) \simeq & \sum_{i=0}^{m_{1}-1} \sum_{j=0}^{m_{2}-1} c_{i, j} T_{i, j}^{1,1}(s, t)+\sum_{i=0}^{m_{1}-1} \sum_{j=0}^{m_{2}-1} d_{i, j} T_{i, j}^{1,2}(s, t) \\
& +\sum_{i=0}^{m_{1}-1} \sum_{j=0}^{m_{2}-1} e_{i, j} T_{i, j}^{2,1}(s, t)+\sum_{i=0}^{m_{1}-1} \sum_{j=0}^{m_{2}-1} l_{i, j} T_{i, j}^{2,2}(s, t) \\
= & F 1^{T} . T 11(s, t)+F 2^{T} . T 12(s, t)+F 3^{T} \cdot T 21(s, t)+F 4^{T} . T 22(s, t) \\
= & {\left[\begin{array}{llll}
F 1^{T} & F 2^{T} & F 3^{T} & F 4^{T}
\end{array}\right] \cdot\left[\begin{array}{c}
T 11(s, t) \\
T 12(s, t) \\
T 21(s, t) \\
T 22(s, t)
\end{array}\right]=F^{T} \cdot T(s, t) }
\end{aligned}
$$

or

$f(s, t) \simeq T^{T}(s, t) \cdot F$,

where $F 1, F 2, F 3$ and $F 4$ can be computed by sampling the function $f(s, t)$ at grid points $s_{i}$ and $t_{j}$ such that $s_{i}=i h_{1}$ and $t_{j}=j h_{2}$, for various values of $i$ and $j$. So we have

$(F 1)_{k}=c_{i, j}=f\left(s_{i}, t_{j}\right)$,

$(F 2)_{k}=d_{i, j}=f\left(s_{i}, t_{j+1}\right)$,

$(F 3)_{k}=e_{i, j}=f\left(s_{i+1}, t_{j}\right)$,

$(F 4)_{k}=l_{i, j}=f\left(s_{i+1}, t_{j+1}\right)$,

where $k=i m_{2}+j$ and $i=0,1, \cdots, m_{1}-1, j=0,1, \cdots, m_{2}-1$. The $4 m_{1} m_{2}$-vector

$F=\left[\begin{array}{llll}F 1^{T} & F 2^{T} & F 3^{T} & F 4^{T}\end{array}\right]^{T}$

is called the 2D-TF coefficient vector.

Let $k(s, t, x, y)$ be a function of four variables on $(\Omega \times \Omega)$. It can be approximated with respect to $2 \mathrm{D}-\mathrm{TF}$ as follows:

$k(s, t, x, y) \simeq T^{T}(s, t) \cdot K \cdot T(x, y)$,

where $T(s, t)$ and $T(x, y)$ are 2D-TF vectors of dimension $4 m_{1} m_{2}$ and $4 m_{3} m_{4}$, respectively and $K$ is a $\left(4 m_{1} m_{2} \times 4 m_{3} m_{4}\right) 2 \mathrm{D}$-TF coefficient matrix. This matrix can be represented as

$K=\left[\begin{array}{llll}K 11 & K 12 & K 13 & K 14 \\ K 21 & K 22 & K 23 & K 24 \\ K 31 & K 32 & K 33 & K 34 \\ K 41 & K 42 & K 43 & K 44\end{array}\right]$ 
where each block of $K$ is an $\left(m_{1} m_{2} \times m_{3} m_{4}\right)$-matrix that can be computed by sampling the function $k(s, t, x, y)$ at grid points $\left(s_{i_{1}}, t_{j_{1}}, x_{i_{2}}, y_{j_{2}}\right)$ such that

$$
\begin{array}{lll}
s_{i_{1}}=i_{1} h_{1}, & i_{1}=0,1, \ldots, m_{1}-1, & h_{1}=\frac{1}{m_{1}} \\
t_{j_{1}}=j_{1} h_{2}, & j_{1}=0,1, \ldots, m_{2}-1, & h_{2}=\frac{1}{m_{2}} \\
x_{i_{2}}=i_{2} h_{3}, & i_{2}=0,1, \ldots, m_{3}-1, & h_{3}=\frac{1}{m_{3}} \\
y_{j_{2}}=j_{2} h_{4}, & j_{2}=0,1, \ldots, m_{4}-1, & h_{4}=\frac{1}{m_{4}} .
\end{array}
$$

Choosing $p=i_{1} m_{2}+j_{1}$ and $q=i_{2} m_{4}+j_{2}$, we get

$$
\begin{aligned}
& (K 11)_{p, q}=k\left(s_{i_{1}}, t_{j_{1}}, x_{i_{2}}, y_{j_{2}}\right), \\
& (K 12)_{p, q}=k\left(s_{i_{1}}, t_{j_{1}}, x_{i_{2}}, y_{j_{2}+1}\right) \text {, } \\
& (K 13)_{p, q}=k\left(s_{i_{1}}, t_{j_{1}}, x_{i_{2}+1}, y_{j_{2}}\right) \text {, } \\
& (K 14)_{p, q}=k\left(s_{i_{1}}, t_{j_{1}}, x_{i_{2}+1}, y_{j_{2}+1}\right) \text {, } \\
& (K 21)_{p, q}=k\left(s_{i_{1}}, t_{j_{1}+1}, x_{i_{2}}, y_{j_{2}}\right), \\
& (K 22)_{p, q}=k\left(s_{i_{1}}, t_{j_{1}+1}, x_{i_{2}}, y_{j_{2}+1}\right) \text {, } \\
& (K 23)_{p, q}=k\left(s_{i_{1}}, t_{j_{1}+1}, x_{i_{2}+1}, y_{j_{2}}\right) \text {, } \\
& (K 24)_{p, q}=k\left(s_{i_{1}}, t_{j_{1}+1}, x_{i_{2}+1}, y_{j_{2}+1}\right) \text {, } \\
& (K 31)_{p, q}=k\left(s_{i_{1}+1}, t_{j_{1}}, x_{i_{2}}, y_{j_{2}}\right), \\
& (K 32)_{p, q}=k\left(s_{i_{1}+1}, t_{j_{1}}, x_{i_{2}}, y_{j_{2}+1}\right) \text {, } \\
& (K 33)_{p, q}=k\left(s_{i_{1}+1}, t_{j_{1}}, x_{i_{2}+1}, y_{j_{2}}\right) \text {, } \\
& (K 34)_{p, q}=k\left(s_{i_{1}+1}, t_{j_{1}}, x_{i_{2}+1}, y_{j_{2}+1}\right) \text {, } \\
& (K 41)_{p, q}=k\left(s_{i_{1}+1}, t_{j_{1}+1}, x_{i_{2}}, y_{j_{2}}\right), \\
& (K 42)_{p, q}=k\left(s_{i_{1}+1}, t_{j_{1}+1}, x_{i_{2}}, y_{j_{2}+1}\right), \\
& (K 43)_{p, q}=k\left(s_{i_{1}+1}, t_{j_{1}+1}, x_{i_{2}+1}, y_{j_{2}}\right) \text {, } \\
& (K 44)_{p, q}=k\left(s_{i_{1}+1}, t_{j_{1}+1}, x_{i_{2}+1}, y_{j_{2}+1}\right) \text {. }
\end{aligned}
$$

In this paper for convergence of the proposed method, we supposed that $m_{1}=m_{2}=m_{3}=m_{4}=m$. More details about the properties of the triangular functions are given in $[5,8]$.

\section{Solving linear 2D-FIES-2}

In this section, we present a 2D-TFs method to solve a linear 2DFIES-2. First consider the following two-dimensional Fredholm integral equations of the second kind (2D-FIE-2):

$u(x, y)=g(x, y)+\lambda \int_{0}^{1} \int_{0}^{1} k(x, y, s, t) u(s, t) \mathrm{d} s \mathrm{~d} t$,

where $k(x, y, s, t)$ is an orbitary kernel function over $(\Omega \times \Omega)$ and $u(x, y)$ and $g(x, y)$ are real valued functions and $u(x, y)$ is unknown. Now, we introduce the 2D-FIES-2 in the following form

$$
\left\{\begin{array}{l}
u_{1}(x, y)=g_{1}(x, y)+\sum_{j=1}^{n} \lambda_{1 j} \int_{0}^{1} \int_{0}^{1} k_{1 j}(x, y, s, t) u_{j}(s, t) \mathrm{d} s \mathrm{~d} t \\
u_{2}(x, y)=g_{2}(x, y)+\sum_{j=1}^{n} \lambda_{2 j} \int_{0}^{1} \int_{0}^{1} k_{2 j}(x, y, s, t) u_{j}(s, t) \mathrm{d} s \mathrm{~d} t \\
\vdots \\
u_{n}(x, y)=g_{n}(x, y)+\sum_{j=1}^{n} \lambda_{n j} \int_{0}^{1} \int_{0}^{1} k_{n j}(x, y, s, t) u_{j}(s, t) \mathrm{d} s \mathrm{~d} t
\end{array}\right.
$$

where $k_{i j}(x, y, s, t), i, j=1, \ldots, n$, are an orbitary kernel function over $(\Omega \times \Omega)$ and $\lambda_{i j} \neq 0, i, j=1, \ldots, n$ are real constants and $u_{i}(x, y)$ and $g_{i}(x, y)$ are real valued functions for $i=1, \cdots, n$ and $u_{1}(x, y), u_{2}(x, y), \ldots, u_{n}(x, y)$ are the solutions to be determined. For convenience, we consider the $i$ th equation of system (12) as

$u_{i}(x, y)=g_{i}(x, y)+\sum_{j=1}^{n} \lambda_{i j} \int_{0}^{1} \int_{0}^{1} k_{i j}(x, y, s, t) u_{j}(s, t) \mathrm{d} s \mathrm{~d} t$.
For solving system (12) by using 2D-TFs, first let us expand $u_{i}(x, y)$, $g_{i}(x, y)$ and $k_{i j}(x, y, s, t)$ by using Eqs. (8) and (10) as follows

$u_{i}(x, y) \simeq T^{T}(x, y) \cdot U_{i}$,

$g_{i}(x, y) \simeq T^{T}(x, y) \cdot G_{i}$,

$k_{i j}(x, y, s, t) \simeq T^{T}(x, y) \cdot K_{i j} \cdot T(s, t)$,

where $U_{i}$ and $G_{i}$ for $i=1, \ldots, n$ are similar to Eq. (9) as follows

$U_{i}=\left[\begin{array}{llll}U 1_{i}^{T} & U 2_{i}^{T} & U 3_{i}^{T} & U 4_{i}^{T}\end{array}\right]^{T}$,

$G_{i}=\left[\begin{array}{llll}G 1_{i}^{T} & G 2_{i}^{T} & G 3_{i}^{T} & G 4_{i}^{T}\end{array}\right]^{T}$

and $K_{i j}$ for $i, j=1, \ldots, n$ are similar to of Eq. (11) as follows

$K_{i j}=\left[\begin{array}{llll}K 11_{i j} & K 12_{i j} & K 13_{i j} & K 14_{i j} \\ K 21_{i j} & K 22_{i j} & K 23_{i j} & K 24_{i j} \\ K 31_{i j} & K 32_{i j} & K 33_{i j} & K 34_{i j} \\ K 41_{i j} & K 42_{i j} & K 43_{i j} & K 44_{i j}\end{array}\right]$.

Substituting the Eqs. (14) into Eq. (13), we get

$$
\begin{aligned}
T^{T}(x, y) U_{i} & \simeq T^{T}(x, y) G_{i} \\
& +\sum_{j=1}^{n} \lambda_{i j} \int_{0}^{1} \int_{0}^{1}\left(T^{T}(x, y) K_{i j} T(s, t) T^{T}(s, t) U_{j}\right) \mathrm{d} s \mathrm{~d} t \\
& =T^{T}(x, y) G_{i} \\
& +T^{T}(x, y) \sum_{j=1}^{n} \lambda_{i j} K_{i j}\left(\int_{0}^{1} \int_{0}^{1} T(s, t) T^{T}(s, t) \mathrm{d} s \mathrm{~d} t\right) U_{j} .
\end{aligned}
$$

Next, by substituting the Eq. (6) into Eq. (15), we can write

$T^{T}(x, y) U_{i} \simeq T^{T}(x, y) G_{i}+T^{T}(x, y) \sum_{j=1}^{n} \lambda_{i j} K_{i j} D U_{j}$

Thus we have

$U_{i}=G_{i}+\sum_{j=1}^{n} \lambda_{i j} K_{i j} D U_{j}$

Then we get the following system

$\sum_{j=1}^{n}\left(\Delta_{i j}-\lambda_{i j} K_{i j} D\right) U_{j}=G_{i}$

where

$\Delta_{i j}=\left\{\begin{array}{cc}I & i=j \\ 0 & i \neq j,\end{array}\right.$

for $i, j=1,2, \ldots, n$ and $I$ is a $4 m^{2} \times 4 m^{2}$ identity matrix. By solving matrix system (16) with Gauss elimination method, we can find $U_{i}$ for $i=1,2, \ldots, n$. So $u_{i}(x, y) \simeq T^{T}(x, y) U_{i}$.

\section{Convergence analysis}

Let $(C[\Omega],\|\|$.$) be a Banach space of all continuous functions on \Omega$ with norm defined by $\|f(x, y)\|=\max _{(x, y) \in \Omega}|f(x . y)|$. We denote the error expression by

$e_{i}(x, y)=\left\|u_{i, m}(x, y)-u_{i}(x, y)\right\|$,

where $u_{i, m}(x, y)$ and $u_{i}(x, y), i=1, \ldots, n$ denote the approximate and exact solutions of the system of two-dimensional Fredholm integral equations, respectively. We'll prove that the present numerical method converges to the exact solution.

Theorem

If $k_{i j}(x, y, s, t), i, j=1,2, \ldots, n$ and $0 \leq x, y, s, t \leq 1$ are bounded and 
continuous, then approximate solution of system (12), converges to the exact solution.

\section{proof}

Suppose that $u_{i, m}(x, y), i=1, \ldots, n$ is an approximate value of the exact solution $u_{i}(x, y)$. Therefore

$$
\begin{aligned}
u_{i, m}(x, y)= & \sum_{p=0}^{m-1} \sum_{q=0}^{m-1} c_{p, q}^{i} T_{p, q}^{1,1}(s, t)+\sum_{p=0}^{m-1} \sum_{q=0}^{m-1} d_{p, q}^{i} T_{p, q}^{1,2}(s, t) \\
& +\sum_{p=0}^{m-1} \sum_{q=0}^{m-1} e_{p, q}^{i} T_{p, q}^{2,1}(s, t)+\sum_{p=0}^{m-1} \sum_{q=0}^{m-1} l_{p, q}^{i} T_{p, q}^{2,2}(s, t),
\end{aligned}
$$

by using Eqs. (13) and (17), we can write

$$
\begin{aligned}
& \left\|u_{i, m}(x, y)-u_{i}(x, y)\right\|=\max _{(x, y) \in \Omega}\left|u_{i, m}(x, y)-u_{i}(x, y)\right| \\
& =\max _{(x, y) \in \Omega} \mid \sum_{j=1}^{n} \lambda_{i j} \int_{0}^{1} \int_{0}^{1} k_{i j}(x, y, s, t)\left(\sum_{p=0}^{m-1} \sum_{q=0}^{m-1} c_{p, q}^{j} T_{p, q}^{1,1}(s, t)\right. \\
& \quad+\sum_{p=0}^{m-1} \sum_{q=0}^{m-1} d_{p, q}^{j} T_{p, q}^{1,2}(s, t)+\sum_{p=0}^{m-1} \sum_{q=0}^{m-1} e_{p, q}^{j} T_{p, q}^{2,1}(s, t) \\
& \left.\quad+\sum_{p=0}^{m-1} \sum_{q=0}^{m-1} l_{p, q}^{j} T_{p, q}^{2,2}(s, t)\right) \mathrm{d} s \mathrm{~d} t \\
& \quad-\sum_{j=1}^{n} \lambda_{i j} \int_{0}^{1} \int_{0}^{1} k_{i j}(x, y, s, t) u_{j}(s, t) \mathrm{d} s \mathrm{~d} t \mid \\
& \leq M \sum_{j=1}^{n} \int_{0}^{1} \int_{0}^{1} \max _{(x, y) \in \Omega}\left|u_{j, m}(s, t)-u_{j}(s, t)\right| \mathrm{d} s \mathrm{~d} t \\
& =M \sum_{j=1}^{n} \int_{0}^{1} \int_{0}^{1}\left\|u_{j, m}(x, y)-u_{j}(x, y)\right\| \mathrm{d} s \mathrm{~d} t
\end{aligned}
$$

where

$$
M=\max _{0 \leq x, y, s, t \leq 1}\left|\lambda_{i j} k_{i j}(x, y, s, t)\right|<\infty .
$$

Also, we have $\lim _{m \rightarrow \infty} u_{j, m}(x, y)=u_{j}(x, y)$, so $\| u_{j, m}(x, y)-$ $u_{j}(x, y) \| \rightarrow 0$ as $m \rightarrow \infty$ for $j=1, \ldots, n$ and since $M$ is bounded, thus

$\lim _{m \rightarrow \infty}\left\|u_{i, m}(x, y)-u_{i}(x, y)\right\| \rightarrow 0$

so the proof is completed.

\section{Numerical illustration}

In this section, we present two examples of linear 2D-FIES-2 and results will be compared with the exact solutions. All results are computed by using a program written in the Matlab R2014a. in this regard, The result presented in the following Tables and Figures.

\section{Example 5.1}

Consider the system of linear two-dimensional Fredholm integral equations

$$
\left\{\begin{aligned}
u_{1}(x, y)= & x y-\frac{6}{20} x+\int_{0}^{1} \int_{0}^{1} x u_{1}(s, t) \mathrm{d} s \mathrm{~d} t+\int_{0}^{1} \int_{0}^{1} x s t^{4} u_{2}(s, t) \mathrm{d} s \mathrm{~d} t \\
u_{2}(x, y)= & x^{2}-\frac{1}{6} x y-\frac{1}{3} y^{2}+\int_{0}^{1} \int_{0}^{1} x y s u_{1}(s, t) \mathrm{d} s \mathrm{~d} t \\
& +\int_{0}^{1} \int_{0}^{1} y^{2} u_{2}(s, t) \mathrm{d} s \mathrm{~d} t
\end{aligned}\right.
$$

One can easily verify that $\left(u_{1}(x, y), u_{2}(x, y)\right)=\left(x y, x^{2}\right)$ is an exact solution of the given problem.

The absolute error of $u_{i}(x, y)$ :

$E_{i}=\left|u_{i, m}(x, y)-u_{i}(x, y)\right|$

for $i=1,2$ with $m=32$, is listed in Table 1 . Also Fig. 1 illustrate the comparison values between the exact solution and the approximate solution by the presented method. Moreover, Absolute error
Table 1: Numerical results for Example 1, with $m=32$.

\begin{tabular}{ccc}
\hline$(x, y)$ & $\begin{array}{c}\text { Absolute error } \\
E_{1}\end{array}$ & $\begin{array}{c}\text { Absolute error } \\
E_{2}\end{array}$ \\
\hline$(0.0,0.0)$ & $0.0000 \mathrm{e}-00$ & $0.0000 \mathrm{e}-00$ \\
$(0.1,0.1)$ & $2.4369 \mathrm{e}-05$ & $1.5985 \mathrm{e}-04$ \\
$(0.2,0.2)$ & $4.8738 \mathrm{e}-05$ & $2.4868 \mathrm{e}-04$ \\
$(0.3,0.3)$ & $7.3107 \mathrm{e}-05$ & $2.6647 \mathrm{e}-04$ \\
$(0.4,0.4)$ & $9.7476 \mathrm{e}-05$ & $2.1324 \mathrm{e}-04$ \\
$(0.5,0.5)$ & $1.2184 \mathrm{e}-04$ & $8.8975 \mathrm{e}-05$ \\
$(0.6,0.6)$ & $1.4621 \mathrm{e}-04$ & $2.8442 \mathrm{e}-04$ \\
$(0.7,0.7)$ & $1.7058 \mathrm{e}-04$ & $4.0883 \mathrm{e}-04$ \\
$(0.8,0.8)$ & $1.9495 \mathrm{e}-04$ & $4.6221 \mathrm{e}-04$ \\
$(0.9,0.9)$ & $2.1932 \mathrm{e}-04$ & $4.4457 \mathrm{e}-04$ \\
\hline
\end{tabular}
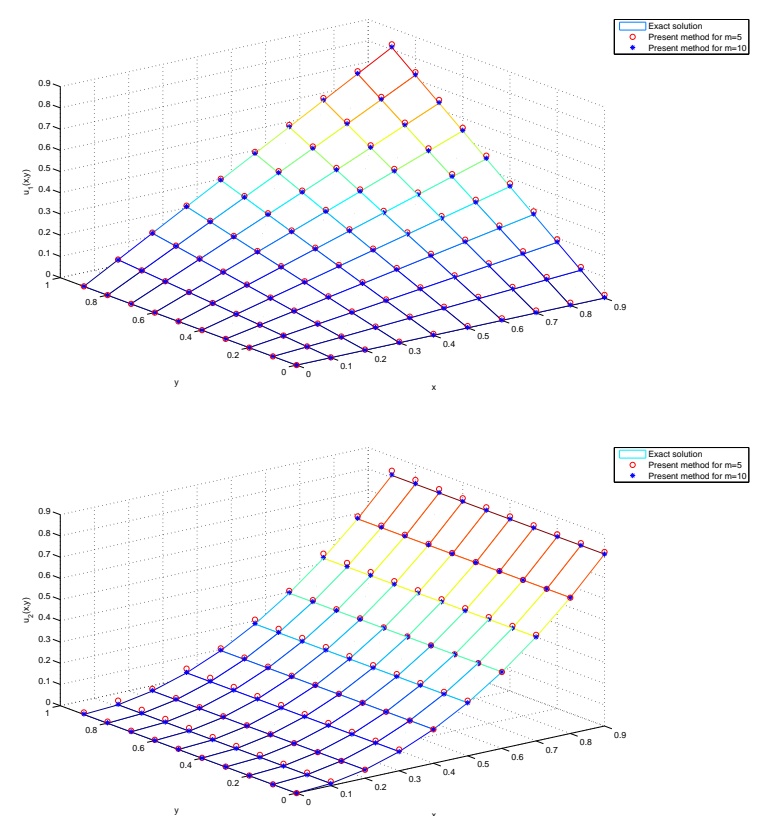

Figure 1: Comparison between the Exact solution and the Approximate solution by the present method for $m=5$ and 10 for Example 1 .

functions obtained by the present method also shown in Figs. 2 and 3.

Example 5.2

Consider the system of linear two-dimensional Fredholm integral equations

$\left\{\begin{array}{l}u_{1}(x, y)=g_{1}(x, y)+\int_{0}^{1} \int_{0}^{1} y e^{s} u_{1}(s, t) \mathrm{d} s \mathrm{~d} t+\int_{0}^{1} \int_{0}^{1} s t u_{2}(s, t) \mathrm{d} s \mathrm{~d} t, \\ u_{2}(x, y)=g_{2}(x, y)+\int_{0}^{1} \int_{0}^{1} u_{1}(s, t) \mathrm{d} s \mathrm{~d} t+\int_{0}^{1} \int_{0}^{1} x u_{2}(s, t) \mathrm{d} s \mathrm{~d} t\end{array}\right.$

where

$g_{1}(x, y)=e^{x}(1+y)-\frac{3}{4} y\left(e^{2}-1\right)-\frac{1}{9}$

$g_{2}(x, y)=x y-\frac{3}{2}(e-1)-\frac{1}{4} x$.

One can easily verify that $\left(u_{1}(x, y), u_{2}(x, y)\right)=\left(e^{x}(1+y), x y\right)$ is an exact solution of the given problem.

The results for Example 2 are shown in Table 2. Also Fig. 4 shows the comparison values between the exact solution and the approximate solution by the presented method for $m=5$ and 10 .

\section{Conclusion}

In this paper, we introduce TFs method for approximating the solution of the linear 2D-FIES-2. The structural properties of TFs 


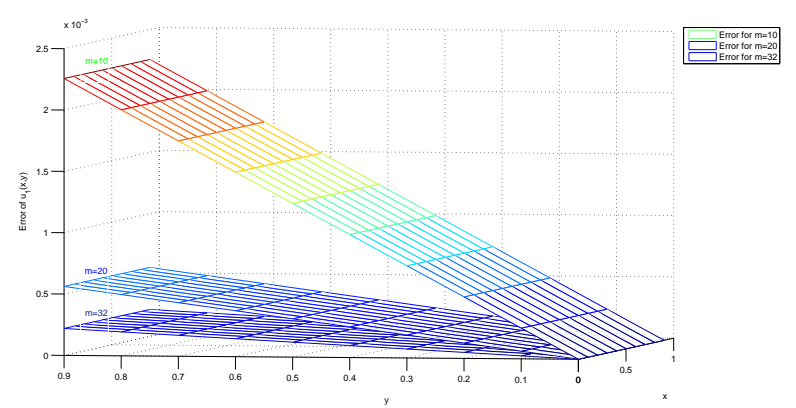

Figure 2: Absolute error $u_{1}(x, y)$ by the present method for $m=10,20,32$ for Example 1.

Table 2: Numerical results for Example 2, with $m=32$

\begin{tabular}{ccc}
\hline$(x, y)$ & $\begin{array}{c}\text { Approximate solution } \\
\left(u_{1, m}, u_{2, m}\right)\end{array}$ & $\begin{array}{c}\text { Absolute error } \\
\left(E_{1}, E_{2}\right)\end{array}$ \\
\hline$(0.0,0.0)$ & $(0.9994,-9.4786 \mathrm{e}-04)$ & $(5.5292 \mathrm{e}-04,9.4786 \mathrm{e}-04)$ \\
$(0.1,0.1)$ & $(1.2151,8.8626 \mathrm{e}-03)$ & $(5.7828 \mathrm{e}-04,1.1374 \mathrm{e}-03)$ \\
$(0.2,0.2)$ & $(1.4651,3.8632 \mathrm{e}-02)$ & $(6.2267 \mathrm{e}-04,1.3270 \mathrm{e}-03)$ \\
$(0.3,0.3)$ & $(1.7541,8.8483 \mathrm{e}-02)$ & $(7.1051 \mathrm{e}-04,1.5166 \mathrm{e}-03)$ \\
$(0.4,0.4)$ & $(2.0877,1.5829 \mathrm{e}-01)$ & $(8.7451 \mathrm{e}-04,1.7061 \mathrm{e}-03)$ \\
$(0.5,0.5)$ & $(2.4719,2.4810 \mathrm{e}-01)$ & $(1.1576 \mathrm{e}-03,1.8957 \mathrm{e}-03)$ \\
$(0.6,0.6)$ & $(2.9143,3.5791 \mathrm{e}-01)$ & $(1.0493 \mathrm{e}-03,2.0853 \mathrm{e}-03)$ \\
$(0.7,0.7)$ & $(3.4224,4.8773 \mathrm{e}-01)$ & $(9.9746 \mathrm{e}-04,2.2749 \mathrm{e}-03)$ \\
$(0.8,0.8)$ & $(4.0049,6.3754 \mathrm{e}-01)$ & $(1.0519 \mathrm{e}-03,2.4644 \mathrm{e}-03)$ \\
$(0.9,0.9)$ & $(4.6720,8.0735 \mathrm{e}-01)$ & $(1.2785 \mathrm{e}-03,2.6540 \mathrm{e}-03)$ \\
\hline
\end{tabular}

are utilized to reduce the 2D-FIES-2 to a linear system of algebraic equations, without using any integration. In the above presented numerical examples one can see that the proposed method well performs for linear 2D-FIES-2.

\section{References}

[1] H. Almasieh, M. Roodaki, “ Triangular functions method for the solution of Fredholm integral equations system", Ain Shams Engineering Journal, (2012), 3, 411-416.

[2] E. Babolian, Z. Masouri and S. Hatamzadeh-Varmazyar, “ A direct method for numerically solving integral equations system using orthogonal triangular functions", Int. J. Industrial Math., (2009), 2, 135145 .

[3] E. Babolian, R. Mokhtari, M. Salmani, "Using direct method for solving variational problems via triangular orthogonal functions", Appl Math Comput, (2007), 191, 206-17.

[4] E. Babolian, Z. Masouri, S. Hatamzadeh-Varmazyar, " Numerical solution of nonlinear Volterra-Fredholm integro-differential equations via direct method using triangular functions", Comput Math Appl., (2009), 58, 239-47.

[5] A. Deb, G. Sarkar, A. Sengupta, “ Triangular orthogonal functions for the analysis of continuous time systems." New Delhi: Elsevier, (2007).

[6] A. Deb, A. Dasgupta, G. Sarkar, “ A new set of orthogonal functions and its application to the analysis of dynamic systems.", J Frank Inst (2006), 343, 1-26.

[7] E. Hengamian Asl, "Solving linear fuzzy Fredholm integral equations system by triangular functions.", Jordan Journal of Mathematics and Statistics, (2016), 9(3), 185-201.

[8] K. Maleknejad, Z. Jafari Behbahani, “ Applications of twodimensional triangular functions for solving nonlinear class of mixed Volterra Fredholm integral equations", Math Comput Model , (2012), $55,1833-44$.

[9] F. Mirzaee, M. Komak Yari, E. Hadadiyan, “ Numerical solution of two-dimensional fuzzy Fredholm integral equations of the second kind using triangular functions", Beni-Suef university journal of basic and appl., (2015), 1-10.

[10] X. Lan, "Variational iteration method for solving integral equations", Comput. Math. Appl., (2007), 54, 1071-78.

[11] F.G. Tricomi, " Integral equations", Dover Publications, New York, (1982).

[12] W. Xie, F. Lin " A fast numerical solution method for two dimensional Fredholm integral equations of the second kind", Appl Numer Math (2009), 59, 1709-19.
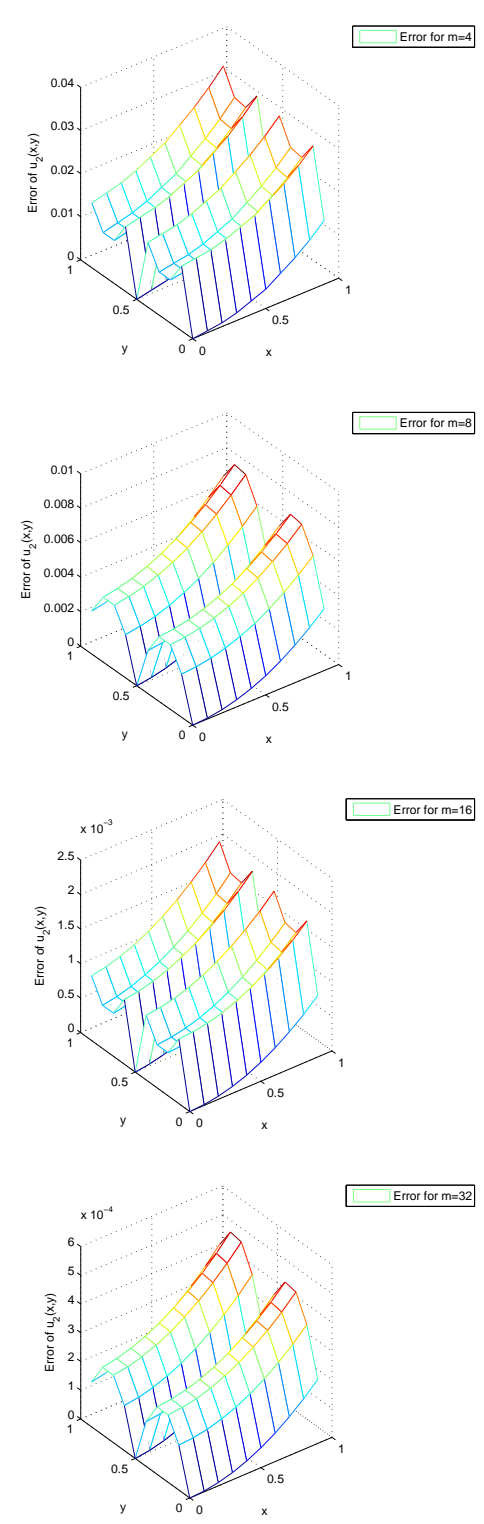

Figure 3: Absolute error $u_{2}(x, y)$ by the present method for $m=4,8,16,32$ for Example 1.

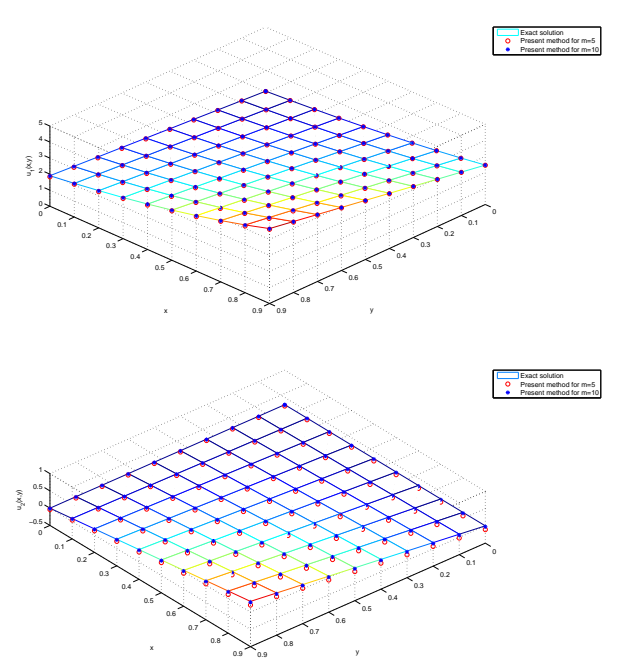

Figure 4: Comparison between the Exact solution and the Approximate solution by the present method for $m=5$ and 10 for Example 2 . 